\title{
Estudio Comparativo de Sulfato de Aluminio y Semillas de Moringa oleifera para la Depuración de Aguas con Baja Turbiedad
}

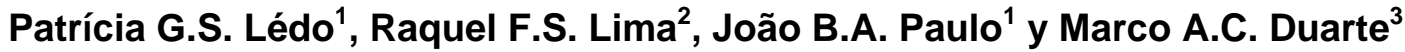 \\ Universidade Federal do Rio Grande do Norte, Centro de Tecnologia, (1) Departamento de \\ Engenharia Química, (2) Departamento de Geologia, Av. Senador Salgado Filho 3000, Campus \\ Universitário, 59072-970-Natal, RN-Brasil \\ (e mail: patiledo@eq.ufrn.br, jbosco@eq.ufrn.br, raquel@geologia.ufrn.br) \\ (3) Compañía de Aguas y Saneamientos de Río Grande do Norte- CAERN/ RN -Brasil \\ (e mail: marcocalazans@oi.com.br)
}

\section{Resumen}

El presente trabajo tiene como objetivo investigar la eficiencia de dos coagulantes, el sulfato de aluminio y semillas de Moringa oleifera, para remoción de turbiedad en muestras del agua provenientes de la Laguna de Jiqui, estado del Rio Grande do Norte, Natal-Brasil. Las dosificaciones y $\mathrm{pH}$ de los coagulantes fueron variados entre límites establecidos. Se realizaron pruebas, en tubos de ensayo Jar-Test y medidas de movilidad electroforética para determinar el potencial Zeta y para determinar los posibles mecanismos involucrados en el proceso de coagulación. Los mecanismos de coagulación con semillas de Moringa oleifera son adsorción y neutralización de cargas y adsorción y formación de puentes. Los resultados sugieren que para aguas de baja turbiedad, las semillas de Moringa oleifera pueden ser un sustituto potencialmente viable al sulfato de aluminio para la clarificación de dichas aguas.

\section{Comparative Study of Aluminum Sulfate and Moringa oleifera seeds as Coagulants in the Clarification of Water with Low Turbidity}

\begin{abstract}
The objective of the present work is to investigate the efficiency of a coagulant prepared from Moringa oleifera seeds, for clarifying water samples with low turbidity from the Jiqui Lake, in the State of Rio Grande do Norte, Natal-Brazil. The dosages and pH of the coagulants were changed within defined limits. Jar-test essays and measurements of electrophoretic mobility were done to determine the Zeta potential and the possible mechanisms involved in the coagulation process. The mechanisms of coagulation with Moringa oleifera are absorption and neutralization of charges and absorption and bridging. The results suggest that for low turbidity waters, Moringa oleifera seeds represent a potentially viable substitute of aluminum sulfate for water clarification.
\end{abstract}

Keywords: clarification of water, coagulation, Zeta potential, natural coagulants, Moringa oleifera 


\section{INTRODUCCIÓN}

Uno de los grandes problemas afrontados por el mundo es la disponibilidad de agua dulce, ya que esta es vital para la supervivencia de los seres humanos. En el planeta existe una limitación de la disponibilidad de agua dulce. Del 0,8\% del agua dulce existente, el $97 \%$ son aguas subterráneas y solamente el $3 \%$ es agua superficial. Aún así, no siempre esta es apropiada para el consumo humano, haciéndose necesario realizar la remoción de las impurezas presentes para adecuarla a los patrones de potabilidad. El color y la turbiedad son los principales parámetros que se consideran en la evaluación de la calidad de efluentes tratados, siendo indicadores sanitarios y patrones de aceptación del agua para consumo humano (FUNASA, 2006). En Brasil, de acuerdo con el decreto $n^{\circ}$ 518/2004 del Ministerio de la salud, el valor máximo de turbiedad permitido es de 5,0 NTU como nivel establecido para consumo humano.

Las partículas coloidales que confieren turbiedad y color, sobre todo en aguas naturales, poseen en su mayor parte cargas eléctricas negativas en su superficie, que crean una barrera repelente entre sí, lo que imposibilita su aglomeración. Así, se hace necesario promover la alteración de las características de la superficie de las partículas con la adición de coagulantes, siendo uno de los primeros pasos en el proceso de tratamiento del agua en la entrada del agua bruta de una Estación de tratamiento de agua. Todas las demás etapas posteriores dependen del éxito de la coagulación.

En este trabajo se investigó la eficiencia de dos coagulantes, el sulfato de aluminio y semillas de Moringa oleifera, para remoción de la turbiedad en muestras del agua con baja turbidez. Se determinó también el potencial Zeta, con el objetivo de ayudar en la comprensión de los mecanismos involucrados en la coagulación, pues indica cambios en el potencial en la superficie y las fuerzas de repulsión entre coloides, como forma de evaluar si parámetros como el $\mathrm{pH}$ y la dosificación de coagulante empleados están coadyuvando a la eficiencia del proceso.

El potencial Zeta $(\zeta)$ es posiblemente una de las pocas magnitudes relacionadas a fenómenos superficiales que pueden ser investigadas directamente por técnicas experimentales muy bien establecidas (Araújo et al., 1990). La determinación del potencial Zeta, en este trabajo, fue realizada usando una técnica llamada microelectroforesis.

Las sales de aluminio son los coagulantes más ampliamente utilizados en el tratamiento del agua y de aguas residuales en todo el mundo. Sin embargo diversos investigadores (Crapper et al., 1973, Martyn et al., 1989, Miller, et al., 1984) sugieren que el aluminio puede inducir la enfermedad de Alzheimer.

A la vista de esta realidad, en años recientes, ha existido un aumento en el interés en el desarrollo de coagulantes naturales alternativos, como el que puede ser extraído de las semillas de Moringa oleifera (MO). Coagulantes naturales de origen vegetal y mineral han sido usados en el tratamiento de aguas antes del advenimiento de productos químicos sintéticos como las sales de aluminio y férricos (Ndabigengesere et al., 1995).

Diversos estudios mostraron que la utilización del Moringa oleifera es comparable al sulfato de aluminio, siendo recomendada su utilización como uno coagulante eficaz para el tratamiento de agua en diversas aplicaciones (Diaz et al., 1999; Jahn, 1986; Ndabigengesere et al., 1995; Ndabigengesere y Narasiah, 1998; Sutherland et al., 1994).

La Moringa oleifera es una planta tropical perteneciente la familia Moringaceae. Es originaria del noroeste de la India, y es conocida en Brasil como Quiabo de quina y Lirio blanco (Lopes y Silva, 2004). El coagulante es declaradamente activo, seguro y barato (Castro y Silva, 2004; Ghebremichael et al., 2005, Ndabigengesere et al., 1995), siendo posible su amplia utilización en el tratamiento del agua, lo que trae beneficios económicos para los países productores, además de constituirse en una alternativa ambientalmente correcta.

Ndabigengesere et al. (1995), han estudiado la eficiencia y las propiedades de MO como coagulante natural y su mecanismo de coagulación en el agua turbia. Verificaron que los componentes activos 
de las semillas de MO son proteínas catiónicas solubles que tienen peso molecular de cerca de 13 $\mathrm{kDa}$ y punto isoeléctrico en $\mathrm{pH}$ entre 10 y 11 , sugieren que el mecanismo predominante de coagulación sea el de adsorción y neutralización de cargas. Ghebremichael et al. (2005), verificaron que las proteínas tienen ponto isoeléctrico 9.6 y masa molecular menos que 6,5 kDa. Galão et al. (2006), realizaron evaluación química y estructúrale de las semientes de Moringa oleifera, observaron que se caracterizan por uno elevado proporción de proteínas, aproximadamente $40 \%$.

Ndabigengesere y Narasiah (1998), verificaron que el empleo de MO no presenta cambios significativos en los valores de $\mathrm{pH}$ y conductividad del agua después de su tratamiento. Según los autores la mayor desventaja en la utilización de la $\mathrm{MO}$ es el incremento del carbono orgánico disuelto en el agua tratada, que aumenta la demanda de cloro y puede llevar la formación de trihalometanos durante la desinfección por ese agente químico. Este incremento representa también una fuente de olor, sabor, entre otros (Okuda et al., 2001b)

Ndabigengesere et al. (1995), verificaron que el lodo generado no es tóxico y presenta un volumen considerablemente menor do que el lodo producido mediante el empleo del sulfato de aluminio. Diversos investigadores verificaron también la presencia de propiedades bactericidas en las semillas da MO (Jahn, 1986; Ghebremichael et al., 2005).

La capacidad de coagulación de las proteínas se ve incrementada cuando se emplean sales inorgánicas en su extracto (Okuda et al., 2001a). Estos autores investigaron la extracción y purificación de los componentes activos de $\mathrm{MO}$ y verificaron que el coagulante extracto con solución salina no aumenta la concentración orgánica residual de la agua después de la coagulación (Okuda et al., 2001a).

En este trabajo se optó por clarificar las aguas provenientes de la Laguna de Jiqui, estado del Rio Grande do Norte, Natal (Brasil), que son de baja turbiedad, con el objetivo de la reducción del turbidez y expandir la discusión sobre la utilización de coagulantes naturales para la depuración de aguas, además de comparar la eficiencia de ambos coagulantes, el sulfato de aluminio y semillas de Moringa oleifera.

\section{MATERIALES Y MÉTODOS}

Toma de muestras

Todas las muestras fueron tomadas en el canal de entrada del agua sin tratar de la Estación de tratamiento de agua del Jiqui (ETA-Jiqui), perteneciente la Compañía de Aguas y Saneamientos de Río Grande do Norte (CAERN/ RN). Actualmente la tecnología de tratamiento en la ETA-Jiqui es la de filtración directa precedida de coagulación con sulfato de aluminio. Tras la colecta, las muestras fueron almacenadas en recipientes de plástico con capacidad para 20 litros, siendo inmediatamente encaminadas para el laboratorio, donde eran realizados los experimentos.

\section{Reactivos utilizados}

Los reactivos utilizados en los ensayos fueron sulfato de aluminio $\left(\mathrm{Al}_{2}\left(\mathrm{SO}_{4}\right)_{3} .14 .3 \mathrm{H}_{2} \mathrm{O}\right)$ al $1 \%$ adquirido por ETA-Jiqui, Natal/RN, y semillas de Moringa oleifera suministradas por Embrapa Tabuleiros Costeiros, Aracaju/SE. Para la corrección del $\mathrm{pH}$ fueron utilizados cal $(0.5 \%)$ y ácido clorhídrico $(0.1 \mathrm{~N})$.

\section{Preparación de la solución coagulante con semillas de Moringa oleifera}

Las semillas secas fueron seleccionadas y peladas manualmente y luego mezcladas con agua destilada en proporción de $25 \mathrm{~g}$ por litro. Esta suspensión fue batida en licuadora durante 15 minutos y filtrada en papel de filtro $(28 \mu \mathrm{m})$, procedimiento adaptado de Lopes y Silva (2004). La dosificación final aproximada de la suspensión es de $25 \mathrm{~g} / \mathrm{L}$, considerando la masa del polvo usada para preparar la solución. 


\section{Ensayos de coagulación y floculación}

En estas pruebas se utilizó sulfato de aluminio y semillas de Moringa oleifera como coagulantes. En cada ensayo se varió la dosificación de coagulante y se controló el pH. Los experimentos realizados con sulfato de aluminio fueron realizados con pHs 5.0, 6.0 y 7.0. En el caso del Moringa oleifera los experimentos se realizaron con $\mathrm{pHs} 6.4,7.0$ y $8.0 . \mathrm{O} \mathrm{pH}$ de la suspención resultante de $\mathrm{MO}$ fue de 6.4 , no necessitando ajuste. La investigación, con $\mathrm{MO}$, en pHs más alcalinos fue realizada para evaluar esta influencia en la eficiencia en el proceso.

Se realizó pruebas en jar test en los ensayos de coagulación y floculación. Las muestras fueron mezcla (misturadas) a $100 \mathrm{rpm}$, durante 2 minutos, durante este tiempo los coagulantes fueron adicionados con el ayuda de una jeringa para llegar a la dosis y $\mathrm{pH}$ requeridos. La medida del $\mathrm{pH}$ realizada en este momento era considerada a del experimento. La velocidad de agitación era entonces disminuida para $50 \mathrm{rpm}$ durante 15 minutos, después los cuales la muestra era decantada durante 15 minutos. El sobrenadante líquido era entonces filtrado en papel de filtro Watman $40 \mu \mathrm{m}$, determinándose la turbiedad remanente en el filtrado.

La muestra utilizada para las medidas de movilidad electroforética (cerca de $20 \mathrm{~mL}$ ) era retirada después los dos minutos de mistura rápida (100 rpm) no Jar test, y inmediatamente transferida para la célula para las medidas.

\section{Movilidad electroforética y potencial Zeta}

Los datos de potencial Zeta fueron obtenidos por la técnica de micro electroforesis utilizando el equipo "Zeta-Meter System 3.0". Este aparato permite la medida de la movilidad electroforética y el cálculo del potencial Zeta. Se utiliza un microscopio estereoscópico de alta calidad para observar las partículas coloidales dentro de una cámara, llamada celda de electroforesis. Electrodos colocados en la cámara son conectados a una fuente de energía por la cual se crea un campo eléctrico. Coloides cargados cambian de posición y sus velocidades y direcciones se relacionan al potencial Zeta. El equipo mide primero la movilidad electroforética de las partículas que se expresa en $\mu \mathrm{ms}^{-1} / \mathrm{Vcm}^{-1}$. El primer término es sencillamente la medida de velocidad, mientras que el segundo expresa la fuerza del campo eléctrico. El potencial Zeta (en mili volts) es calculado a partir de la movilidad electroforética según la ecuación de Helmoholtz- Smoluchowski (Hunter, 1981):

$$
\zeta=112890 / E_{r} \eta V / E
$$

$\mathrm{u}_{\mathrm{E}}=\mathrm{V} / \mathrm{E}$

Sustituyendo (2) en (1)

$\zeta=112890 / E_{r} \quad \eta \quad u_{E}$

Donde:

$\zeta=$ Potencial Zeta $(\mathrm{mv})$

$\mathrm{E}_{\mathrm{r}}=$ Constante dieléctrica del solvente, dependiendo de la temperatura

$\eta=$ Viscosidad de la solución (poise)

$\mathrm{u}_{\mathrm{E}}=$ Movilidad electroforética $\left(\mu \mathrm{ms}^{-1} / \mathrm{Vcm}^{-1}\right)$

$\mathrm{V}=$ Velocidad promedio de las partículas $(\mu \mathrm{m} / \mathrm{s})$

$E=$ Intensidad del campo eléctrico, definido como U/L

Cada medida fue realizada 15 veces y se consideraron los resultados cuya desviación patrón fue inferior a 4. Los experimentos fueron realizados en el Laboratorio de Tensoactivos del Departamento de Química de la Universidad Federal de Rio Grande do Norte (UFRN). 


\section{Análisis Fisicoquímicos}

Las medidas de turbiedad y $\mathrm{pH}$ fueron realizadas con turbidímetro marca Policontrol modelo AP 2000 y medidor de $\mathrm{pH}$ digital marca Tecnal modelo 3MP, respectivamente. La turbidez inicial es esa medida en el día de la realización del experimento, entonces después de la tomada del agua en la estación, mientras que la turbiedad remanente es esa medida al final de los experimentos en jar test.

\section{RESULTADOS Y DISCUSIÓN}

Sulfato de aluminio

Los resultados de potencial Zeta y turbiedad remanente de las pruebas realizadas en tres valores diferentes de $\mathrm{pH}(5.0,6.0$ y 7.0$)$ son presentados en la figura 1 ( $a, b$ y c).

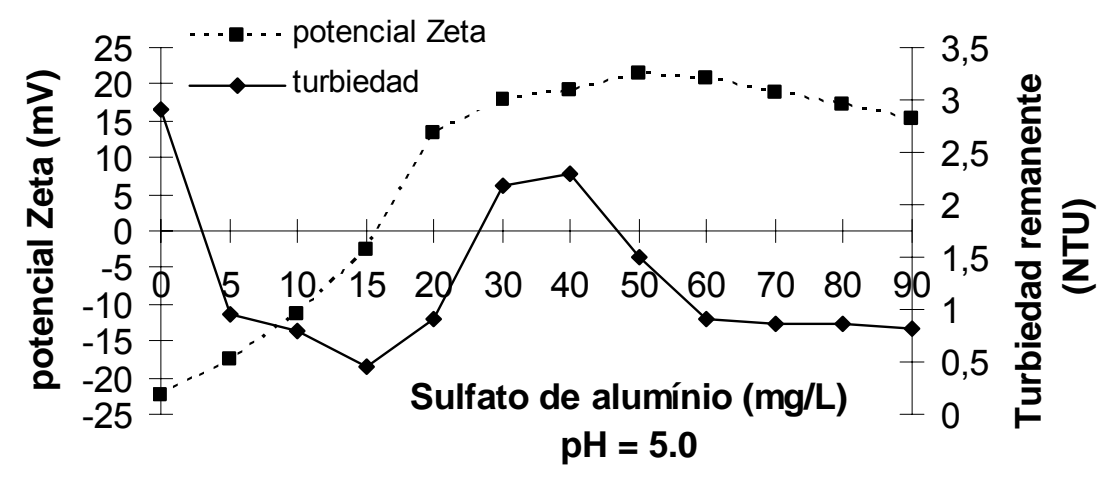

a
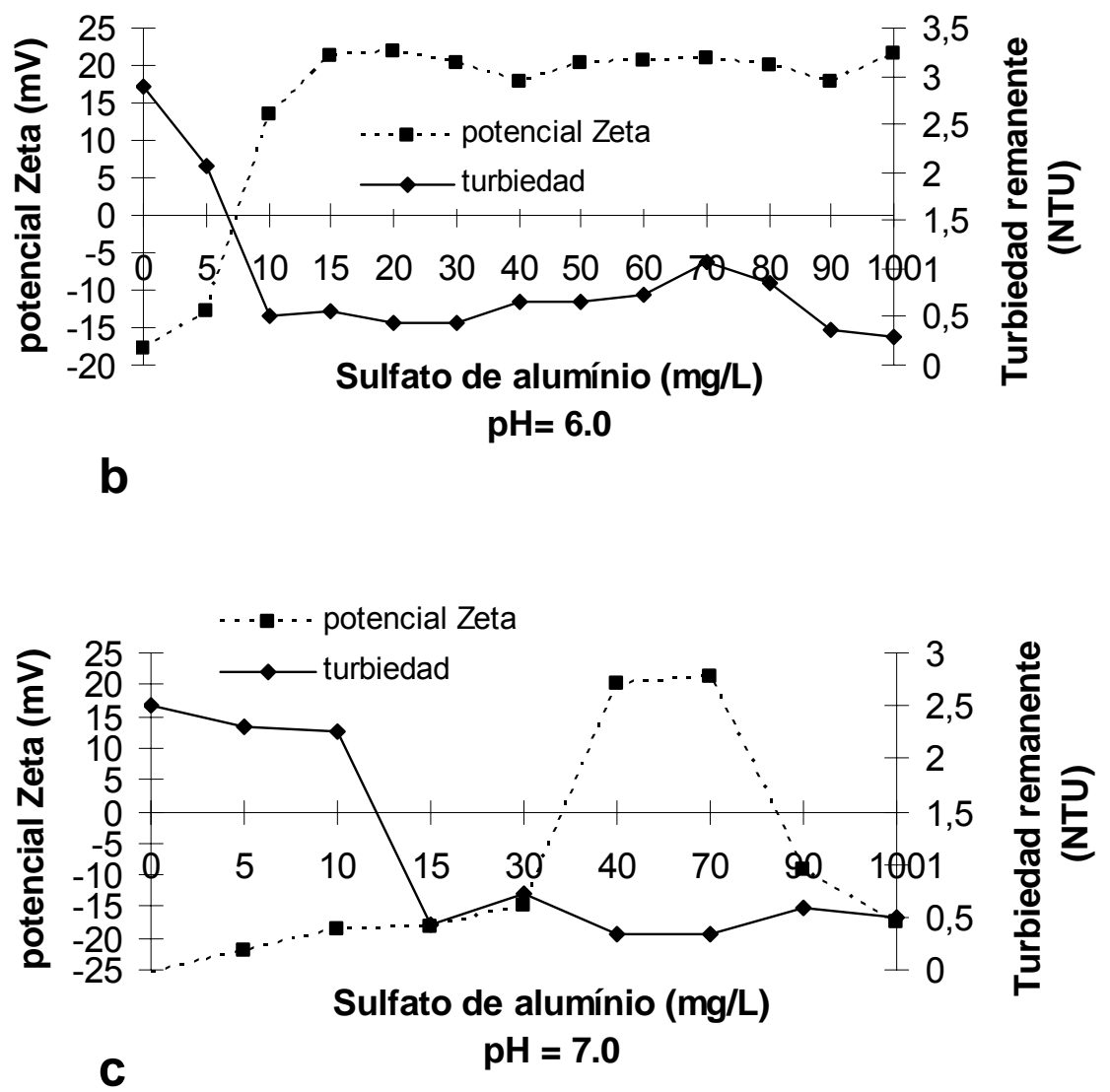

Fig. 1: Potencial Zeta y turbiedad remanente para diferentes dosificaciones de sulfato de aluminio $(\mathrm{pH}=5.0,6.0$ y 7.0$)$. 
En la figura 1.a se muestran, para $\mathrm{pH}=5.0$, las variaciones de las dosificaciones de Sulfato de aluminio y el efecto de estas sobre el potencial Zeta y en la eficiencia de remoción de turbiedad. El potencial Zeta varió entre $-22.50 \mathrm{mV}$ y $+21.57 \mathrm{mV}$, aumentando con el aumento de la dosificación del coagulante. El punto isoeléctrico y la reversión de la carga coloidal son observados en la dosificación aproximada de $16 \mathrm{mg} / \mathrm{L}$. Las observaciones microscópicas durante las medidas realizadas, revelan la presencia de pequeños copos dispersos en medio para todas las dosificaciones comprobadas.

La mejor eficiencia de remoción fue observada en la dosificación de $15 \mathrm{mg} / \mathrm{L}$; correspondiendo a un porcentual de remoción del $84 \%$. Se observa en la figura 1.a que la mejor eficiencia de remoción está próxima al punto donde el potencial Zeta es nulo (punto isoeléctrico). A la vista de estos resultados y considerando la característica de los copos formados, se sugiere que el mecanismo de coagulación predominante en este $\mathrm{pH}$ es el de adsorción-neutralización de cargas. En este mecanismo ocurre la neutralización de la carga de las partículas coloidales por los iones hidrolizados de aluminio, que son adsorbidas en la superficie de los coloides.

La figura 1.b muestra los resultados obtenidos en el $\mathrm{pH}=6.0$. Se observa en la curva del potencial Zeta, el punto isoeléctrico en la dosificación aproximada de $7 \mathrm{mg} / \mathrm{L}$ de coagulante y la reversión de la carga coloidal. El potencial Zeta varió entre -17.90 y $+22.94 \mathrm{Mv}$. Las observaciones microscópicas durante las medidas de movilidad electroforética revelaron la formación de pequeños copos dispersos en medio hasta la dosificación de $80 \mathrm{mg} / \mathrm{L}$. Por encima de esta dosificación se observó la formación de copos más voluminosos y agrupados. La franja de dosificación óptima del coagulante fue de 10 a 30 mg/L, con variaciones de remoción de turbiedad entre 81 y $85 \%$. Tras la reversión de la carga coloidal, los valores de la turbiedad continúan reducidos, sugiriendo que la remoción de turbiedad no está controlada predominantemente por fuerzas electrostáticas. Los resultados de las medidas de ME y la característica de los copos formados sugieren la predominancia del mecanismo de adsorción-neutralización de cargas para bajas dosificaciones de sulfato, pudiendo haber una combinación de los mecanismos de adsorción neutralización de cargas y barredura hasta la dosificación de $80 \mathrm{mg} / \mathrm{L}$. Por encima de la dosificación de $80 \mathrm{mg} / \mathrm{L}$, se verificó la formación de copos mayores y una mejora en la remoción de turbiedad. En esta franja, se sugiere la predominancia del mecanismo de barredura, en el cual aparece la formación de precipitados de hidróxidos de aluminio, juntamente con las partículas coloidales aprisionadas en los mismos (Lacerda, 1997; Pavanelli, 2001).

En la figura 1.c se muestran las variaciones del Potencial Zeta en diferentes dosificaciones de sulfato de aluminio, realizadas en $\mathrm{pH}=$ 7.0. Se verificó que el Potencial Zeta varió entre -25.40 y $+21.37 \mathrm{mV}$, hasta la dosificación de $70 \mathrm{mg} / \mathrm{L}$. Las mejores eficiencias de remoción se observó en las dosificaciones entre 15 a $70 \mathrm{mg} / \mathrm{L}$, correspondiendo a una variación de remoción entre 79 y $86 \%$. Las características de los copos formados observados en el microscopio (copos mayores que los observados en el mecanismo adsorción-neutralización de cargas), sugieren la predominancia del mecanismo de barredura. En las dosificaciones por encima de $80 \mathrm{mg} / \mathrm{L}$ se observó un potencial Zeta negativo. Se sabe que en la coagulación con sulfato de aluminio, las reacciones de hidrólisis forman diferentes compuestos, dependiendo del $\mathrm{pH}$ de origen del agua, alcalinidad, temperatura, otras partículas, materia orgánica, dosificación de coagulante y $\mathrm{pH}$ de coagulación. Es probable que el potencial Zeta negativo observado en las dosificaciones por encima de $80 \mathrm{mg} / \mathrm{L}$, sea debida a los complejos hidroximetálicos presentes en esta dosificación y pH.

Por medio de los resultados presentados en las figuras se observa que el mecanismo de adsorción neutralización de cargas predomina len las bajas dosificaciones de sulfato de aluminio, mientras que el mecanismo de barredura predomina en mayores dosificaciones de coagulante. Diversos investigadores presentan resultados similares, pudiéndose citar: Lacerda (1997), Pavanelli (2001), Teixeira (2003), entre otros.

\section{Moringa oleifera}

Los resultados de potencial Zeta y turbiedad remanente de las pruebas realizadas en tres valores diferentes de $\mathrm{pH} 6.4$ (sin ajuste), 7.0 y 8.0 son presentados en la figura 2 (a, b y c). 


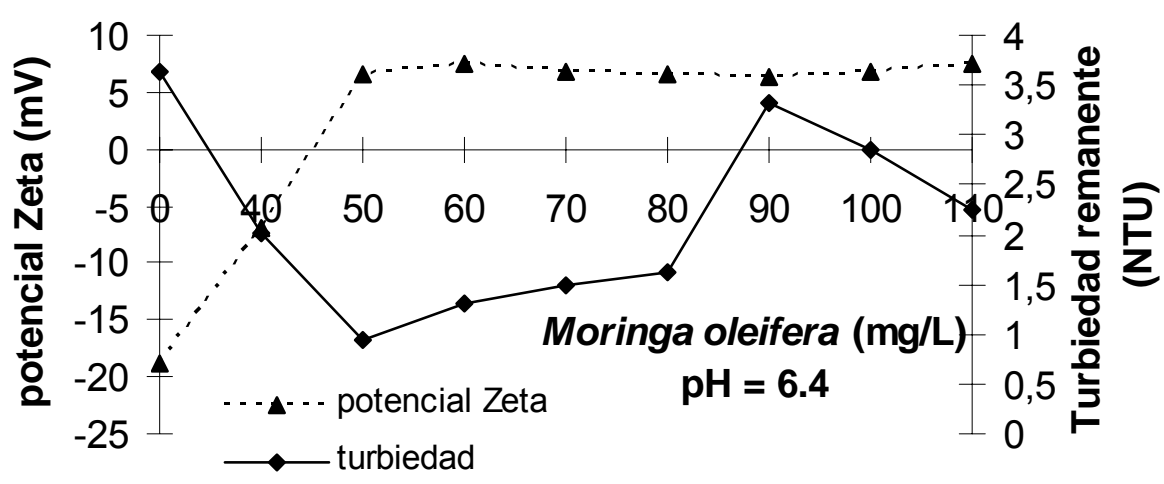

a
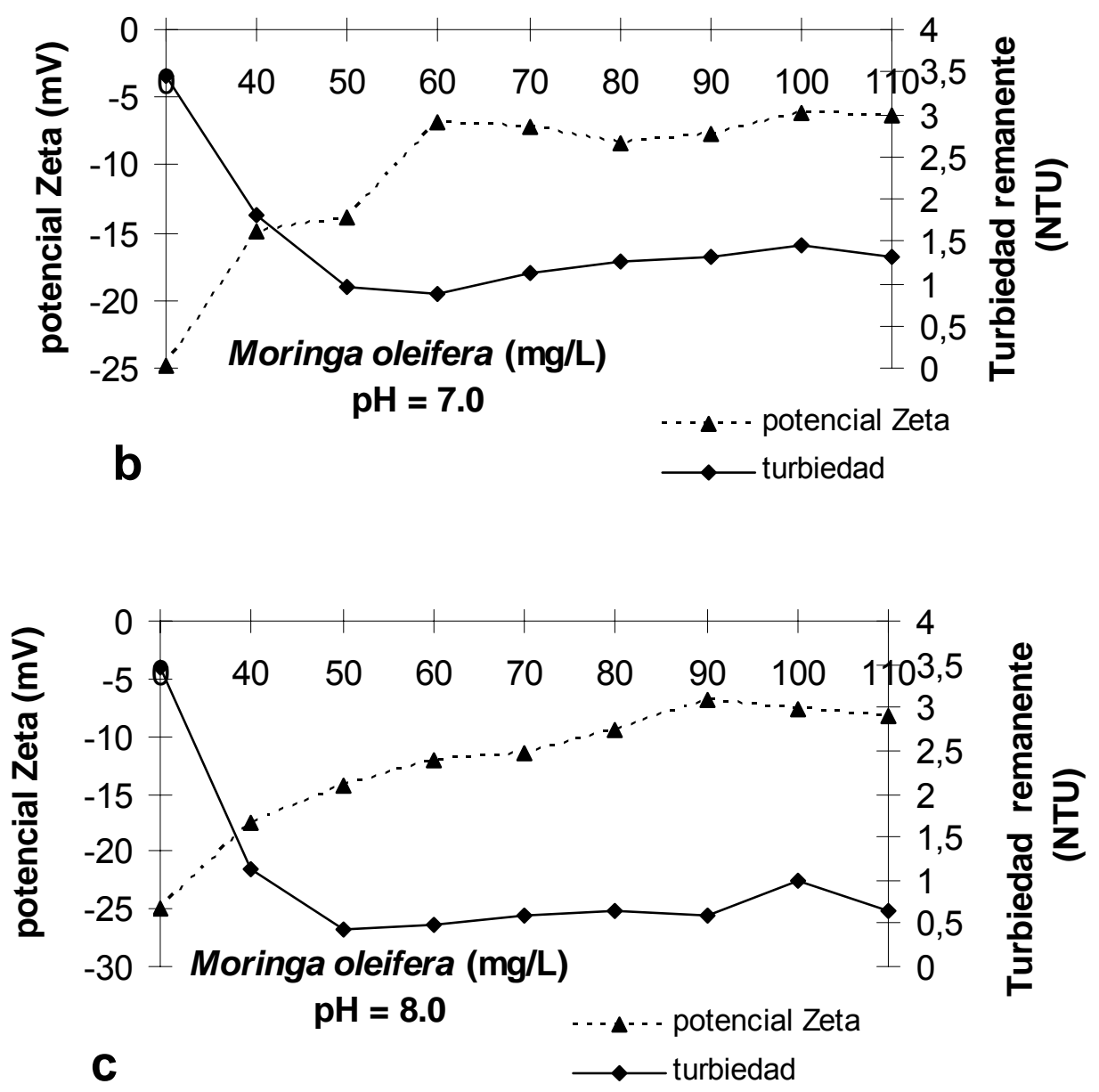

Fig. 2: Potencial Zeta y turbiedad remanente para diferentes dosificaciones de Moringa oleifera $(\mathrm{pH}=6.4,7.0$ y 8.0$)$.

En la figura 2 se muestran las variaciones en la dosificación de Moringa oleifera y su influencia sobre el potencial Zeta y en la eficiencia de remoción de turbiedad. Se observa en la curva del potencial Zeta el paso por el punto isoeléctrico y la reversión en la carga coloidal, que en términos de potencial varió entre -24.90 y $+7.40 \mathrm{mV}$. Se verificó la formación de copos de tamaño pequeño, casi no visibles a simple vista, menores que los observados utilizando el sulfato de aluminio. La mejor eficiencia de remoción fue observada en la dosificación de $50 \mathrm{mg} / \mathrm{L}$, próxima al punto donde el potencial Zeta es nulo, correspondiendo a un porcentual de remoción de turbiedad del 74\%. Las observaciones microscópicas durante las medidas de ME revelaron la formación de poquísimos copos dispersos en 
el medio y en todas las dosificaciones comprobadas. A la vista de los resultados y considerando las características de los copos formados en el presente trabajo, se sugiere que el mecanismo de coagulación predominante es el de adsorción-neutralización de cargas, pudiendo ocurrir simultáneamente el mecanismo de adsorción y formación de puentes.

La figura 2.b muestra los resultados obtenidos con $\mathrm{pH}=7.0$. Se observa que el potencial Zeta varía entre -24.90 y $-6.30 \mathrm{mV}$, y no aparece reversión de la carga coloidal. Las observaciones microscópicas durante las medidas de movilidad electroforética revelaron la formación de una mayor cantidad de copos que con $\mathrm{pH}=6,4$ (sin ajuste). La dosificación óptima de coagulante fue de 60 $\mathrm{mg} / \mathrm{L}$; correspondiendo a un porcentual de remoción del $74 \%$.

En la figura 2.c se muestran las variaciones del Potencial Zeta en las dosificaciones de Moringa oleifera en $\mathrm{pH}=8.0$. Se verificó que el Potencial Zeta varió entre -24.9 y $-8.19 \mathrm{mV}$, no apareciendo reversión de la carga coloidal. La mejor eficiencia de remoción se tuvo con una dosificación de $50 \mathrm{mg} / \mathrm{L}$, con porcentual de remoción del $88 \%$. En este $\mathrm{pH}$ se observa la formación de mayor cantidad de copos que en los pH anteriores. A la vista de los resultados de las medidas de ME, en los pH 7.0 y 8.0, se verifica que la coagulación con semillas de Moringa oleifera no es controlada por fuerzas electrostáticas, lo que nos lleva a sugerir que el mecanismo de coagulación sea el de adsorción y formación de puentes. Ndabigengesere et. al. (1995) verificaron con medidas del potencial Zeta que los mecanismos de coagulación más probables con la utilización de las semillas de Moringa oleifera son los de adsorción-neutralización de cargas y adsorción y formación de puentes, pudiendo incluso ocurrir de forma simultánea.

Ndabigengesere et al. (1995), compararon el rendimiento de los coagulantes sulfato de aluminio e Moringa oleifera y verificaron que los dos coagulantes presentaron dosis óptimos próximas a 50 $\mathrm{mg} / \mathrm{L}$. De acuerdo con Schwarz (2000), para aguas con turbiedad a bajo de 50 NTU la banda de dosis de semillas de MO empleado es de $10-50 \mathrm{mg} / \mathrm{L}$. En este trabajo verificó-se que para la concentración aproximada de $50 \mathrm{mg} / \mathrm{L}$ de $\mathrm{MO}$ existe la situación de máxima disminución de turbiedad para los tres $\mathrm{pH}$, lo que está indicando que el proceso de coagulación no se ve alterado por el pH inicial del medio; dicho de otro modo, el proceso de coagulación es independiente del pH. Este es un dato muy interesante desde el punto de vista para su aplicación directa en una planta de tratamientos de aguas, ya que no se debe corregir el $\mathrm{pH}$ del agua al ingreso del proceso.

Adicionalmente, observó-se que con el aumento del valor del pH, la eficiencia de remoción de turbiedad aumentó alcanzando valores de 74 a $88 \%$ de remoción (Fig. 2).

\section{CONCLUSIONES}

Para el sulfato de aluminio, el mecanismo de adsorción-neutralización de cargas, predomina a bajos $\mathrm{pH}$ mientras que en el $\mathrm{pH}$ 7.0, el mecanismo predominante es el de barredura;

Para las semillas de Moringa oleífera se verificó que la eficiencia de remoción de turbiedad é ligeramente inferior a observada con o sulfato de aluminio, o uso de la primera puede justificarse por el hecho de representar una tecnología ambientalmente correcta. Los mecanismos de coagulación predominantes son los de adsorción-neutralización de cargas y adsorción y formación de puentes;

Este trabajo indica que la utilización del Moringa oleífera es comparable al sulfato de aluminio, pudiendo ser utilizada como coagulante eficaz para la depuración de agua con baja turbiedad.

\section{AGRADECIMIENTOS}

A CAPES, al programa de post graduación de Ingeniería Química-UFRN (PPGEQ), al Laboratorio de Tensoactivos del Departamento de Química-UFRN, a EMBRAPA Tabuleiros Costeiros y CAERN.

\section{REFERENCIAS}

Araújo, A.C., A.E.C.Peres y P.R.G. Brandão; Determinação do Potencial Zeta. In: Simpósio Epusp de Caracterização Tecnológica na Engenharia e Indústria Mineral. Anais: 231-247 São Paulo (1990). 
Castro, F.J.F y F.J.A. Silva; Moringa Oleifera na Melhoria da Qualidade de Efluentes de UASB e de Lagoa de Maturação-Remoção de Cor e Turbidez. In: XI SILUBESA - Simpósio Luso-Brasileiro de Engenharia Sanitária e Ambiental, Natal/RN (2004).

Crapper D.R., S.S. Krishnan y A.J. Dalton; Brain Aluminum Distribution In Alzheimer's Disease And Experimental Neurofibrillary Degeneration. Science: 180, 511-513 (1973).

Diaz, A. y otros cinco autores; Turbidity removal by natural coagulants indigenous to Venezuela. Process Biochemistry: 35, 391-395 (1999).

FUNASA; Manual prático de análise de água. 2edição, 39-79. Fundação Nacional de Saúde, Brasília (2006).

Galão, M.I., L.F. Damasceno y E.S. Brito; Avaliação química e estrutural da semente de moringa, Revista Ciência Agronômica: 37(1), 106-109 (2006).

Ghebremichael, K.A., y otros cuatro autores; $A$ simple purification and activity assay of the coagulant protein from Moringa oleifera seed. Water Research: 39, 2338-2344 (2005).

Hunter, R.J.; Zeta Potential in Colloid Science- Principles and Applications, 59-121. Academic Press, New York (1981).

Jahn, S.A.A.; Proper use of African Natural Coagulants for Rural Water Supplies- Research in the Sudan and a Guide for New Projects, Eschborn, GTZ, Germany (1986).

Lacerda, M.R.S.; A Influência do pH de Coagulação e do Tempo de Floculação na Flotação por ar Dissolvido no Tratamento de Água com Baixa Turbidez e Presença de Algas. Tesis de Magister, Departamento de Engenharia Civil e Ambiental, UNB, Brasília, Brasil (1997).

Lopes, A. S. y F.J.A. Silva;Tratamento de Efluente de Tanque de Piscicultura com Moringa oleifera. In: XI SILUBESA - Simpósio Luso-Brasileiro de Engenharia Sanitária e Ambiental, Natal/RN (2004).

Martyn, C.N. y otros cuatro autores; Geographical Relation Between Alzheimer's Disease and Aluminium in Drinking Water. Lancet: 1, 59-62 (1989).

Miller, R.G. y otros cuatro autores; The Occurrence of Aluminum in Drinking Water. J. Am. Wat. Wks Assoc.: 76, 84-91 (1984).

Ndabigengesere A., K.S. Narasiah y B.G.Talbot; Active Agents and Mechanism of Coagulation of Turbid Waters Using Moringa oleifera. Water Research: 29 (2), 703-710 (1995).

Ndabigengesere, A. y, K.S Narasiah; Quality of Water Treated by Coagulation Using Moringa oleifera Seeds. Water Research: 32(3), 781-791 (1998).

Okuda, T., A.U. Baes, W. Nishijima y M. Okada; Isolation and Characterization of Coagulant Extracted from Moringa oleifera Seed by Salt Solution. Water Research: 35(2), 405-410 (2001a).

Okuda, T., A.U. Baes, W. Nishijima y M. Okada; Coagulation mechanism of salt solution- extracted active component in moringa oleifera seeds. Water Research: 35(3), 830-834 (2001b).

Pavanelli, G.; Eficiência de Diferentes Tipos de Coagulantes na Coagulação, Floculação e Sedimentação de Água com Cor ou Turbidez Elevada. Tesis de Magister, Escola de Engenharia de São Carlos, USP, São Carlos, Brasil (2001).

Schwarz, D.; Water clarification using Moringa oleifera. Gate information service, Eschborn, Germany (2000). 
Sutherland, J.P., G.K. Folkard, M.A.Mtawali y W.D Grant; Moringa oleifera as a Natural Coagulant. In: Proc. of the 20 th WEDC Conference, Affordable Water Supply and Sanitation: 297-299, Colombo, Sri Lanka (1994).

Teixeira, P.C.; Emprego da Flotação Por Ar Dissolvido no Tratamento de Efluentes de Lavagem de Veículos Visando a Reciclagem da Água. Tesis de Magister, Faculdade de Engenharia Civil, Universidade Estadual de Campinas, Campinas, Brasil (2003). 